\title{
Effect of idebenone on bone marrow mesenchymal stem cells in vitro
}

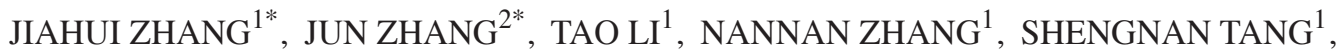

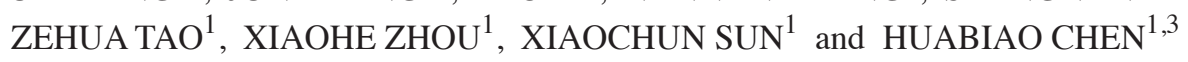 \\ ${ }^{1}$ School of Medicine, Jiangsu University, Zhenjiang, Jiangsu 212001; ${ }^{2}$ Clinical Laboratory, The Affiliated \\ Hospital of Yangzhou University, Yangzhou, Jiangsu 225001, P.R. China; ${ }^{3}$ Vaccine and Immunotherapy \\ Center, Massachusetts General Hospital and Harvard Medical School, Boston, MA 02114, USA
}

Received July 19, 2017; Accepted January 12, 2018

DOI: $10.3892 / \mathrm{mmr} .2018 .8506$

\begin{abstract}
In recent years, stem cell research has continued to benefit from its crossover with chemistry, particularly the investigation of small molecular drugs modulating specific targets to regulate stem cell fate. Idebenone (IDB) is a yellow crystalline powder that is used in the treatment of chronic cerebrovascular diseases. The objective of the present study was to examine whether IDB had an influence on bone marrow-derived mesenchymal stem cells (BMSCs) extracted from the bone marrow of Sprague-Dawley rats. The effects of IDB on cell proliferation, cell cloning and migration were investigated. Cell cycle, apoptosis, DAPI nuclear staining and senescence-associated $\beta$-galactosidase (SA- $\beta$-gal) staining were also examined. The results revealed that IDB at suitable concentrations enhanced cell cloning capacity, promoted the proliferation of BMSCs, delayed cellular senescence, and inhibited cell apoptosis and migration. Western blot analysis indicated that IDB increased the expression of B-cell lymphoma 2 (Bcl-2), signal transducer and activator of transcription-3, Nanog, octamer-binding transcription factor 4, E-cadherin, proliferating cell nuclear antigen, cyclinD1 and cyclinD3, and decreased the expression of Bcl-2-associated X protein, cleaved caspase-3, N-cadherin, vimentin and $\alpha$-smooth muscle actin. In conclusion, these experiments confirmed that IDB in low doses had no toxic effect and may exert protective effects on BMSCs.
\end{abstract}

Correspondence to: Professor Xiaochun Sun, School of Medicine, Jiangsu University, 301 Xuefu Road, Zhenjiang, Jiangsu 212001, P.R. China

E-mail: xiaochun@ujs.edu.cn

Professor Huabiao Chen, Vaccine and Immunotherapy Center, Massachusetts General Hospital and Harvard Medical School, 149 13th Street, Charlestown, Boston, MA 02114, USA

E-mail: huabiao.chen@mgh.harvard.edu

*Contributed equally

Key words: mesenchymal stem cells, idebenone, biological characteristics

\section{Introduction}

IDB is a novel analog of coenzyme Q10 which is widely used for treatment of cardiovascular diseases $(1,2)$. IDB can effectively inhibit lipid peroxidation in brain tissues, mitochondrial membranes and nerve cells, thus protecting cells and mitochondria from oxidative damage. It is well tolerated and has low toxicity (3). IDB has shown significant benefits in neurodegenerative disorders including Alzheimer's disease and hereditary optic neuropathy $(4,5)$. IDB therapy has been applied in Friedreich ataxia patients where it was demonstrated to improve cardiac and neurological functions and slow the progression of the disease (6-14).

Mesenchymal stem cells (MSCs) are important members of the stem cell family which derive during early development of mesoderm and ectoderm. MSCs from a class of undifferentiated cells that constantly regenerate themselves and secrete a large number of cytokines to promote the growth of other stem cells (15). Research has indicated that MSCs are multi-potent stem cells that can differentiate into mature cells including osteoblasts, adipose cells, cartilage cells, muscle cells and nerve cells (16). MSCs can be obtained from many tissues including adult human bone marrow, rat bone marrow, adipose tissue, cord blood and umbilical cord (17-20).

To the best of our knowledge, the efficacy of IDB on bone marrow-derived mesenchymal stem cells (BMSCs) has not been tested. In this study, we aimed to verify whether IDB has an effect on BMSCs and to evaluate the safety and stability of the drug. We thus examined the impact of IDB on the biological characteristics of BMSCs and its potential mechanisms relating to cell apoptosis and cell migration.

\section{Materials and methods}

Isolation and characterization of BMSCs. BMSCs were isolated and cultured according to standard protocols (21). BMSCs were isolated from 80-100 g Sprague-Dawley rats. The cells were collected by flushing the rat femurs with phosphate-buffered saline (PBS) and culturing them in low-glucose Dulbecco's modified Eagle's medium (L-DMEM; Gibco; Thermo Fisher Scientific, Inc., Waltham, MA, USA) containing $10 \%$ (v/v) fetal bovine serum (FBS; Gibco; Thermo 
Fisher Scientific, Inc.), penicillin (100 U/ml; Shandong Lunan Pharmaceutical Factory) and streptomycin (100 U/ml; Shandong Lunan Pharmaceutical Factory) in a humidified atmosphere of $5 \% \mathrm{CO}_{2}$ at $37^{\circ} \mathrm{C}$. The culture medium was replaced at 3-day intervals. The adherent cells were trypsinized with $0.25 \%$ trypsin-EDTA (Invitrogen; Thermo Fisher Scientific, Inc.) and subcultured in new flasks for further expansion when they reached $80-90 \%$ confluence. Cells in passage 3-5 were used for experiments. The experimental protocol was approved by the Institutional Animal Care Committee of Jiangsu University, Zhenjiang, China.

Multi-differentiation capacity. Adipogenesis: The BMSCs $\left(2 \times 10^{4}\right)$ were seeded in 24-well plates in L-DMEM with $10 \%$ FBS. The medium was replaced with adipogenic induction medium (Cyagen Biosciences, Inc., Santa Clara, CA, USA) when cells attained 70-80\% confluency. After cultivation for 21 days, adipogenic differentiation was assessed by the presence of intracellular lipid vesicles stained with Oil Red O (Cyagen Biosciences, Inc.) and visualized using an inverted microscope (TE300; Nikon Corporation, Tokyo, Japan).

Osteogenesis: BMSCs were plated in six-well plates at $1 \times 10^{4}$ cells $/ \mathrm{cm}^{2}$ in L-DMEM with $10 \% \mathrm{FBS}$. After incubation overnight at $37^{\circ} \mathrm{C}$ with $5 \% \mathrm{CO}_{2}$, cells were cultured in osteogenic induction medium (Cyagen Biosciences, Inc.). After treatment for 14 days, osteogenic differentiation was detected by alkaline phosphatase staining following the manufacturer's instructions (Shanghai Sun Biotechnology Co., Ltd, Shanghai, China).

Flow cytometry. The phenotypes of BMSCs were analyzed with flow cytometry using a FACSCalibur flow cytometer (BD Biosciences, Franklin Lakes, NJ, USA) after they were trypsinized with $0.25 \%$ trypsin-EDTA and stained with PE-conjugated monoclonal antibodies against CD29, CD90, CD105 or CD19 (FITC-conjugated) for $30 \mathrm{~min}$ at $4^{\circ} \mathrm{C}$. FITC-IgG1 and PE-IgG1 (BD Biosciences) isotypic immunoglobulins were used as isotype controls.

Proliferation assay. The proliferative ability of BMSCs was determined with 3-(4, 5-dimethylthiazol-2-yl)-2,5-diphenyltetrazolium bromide (MTT) assay. Cells were seeded in 96-well plates at 3,000 cells per well and allowed to attach overnight at $37^{\circ} \mathrm{C}$ with $5 \% \mathrm{CO}_{2}$. Thereafter, cells were treated with different concentrations of IDB (0.5 to $60 \mu \mathrm{g} / \mathrm{ml})$ for $0-96 \mathrm{~h}$. MTT $(20 \mu \mathrm{l})$ was added to each well for the last $4 \mathrm{~h}$. The culture medium was then discarded and $150 \mu 1$ of dimethyl sulfoxide (DMSO) were added to the plates to terminate the reaction. The plates were observed under a microscope after shaking for $10 \mathrm{~min}$ to solubilize the purple formazan crystals. The absorbance values were read at $490 \mathrm{~nm}$ using an enzyme-linked immunosor-bent plate assay reader (FLX800; BioTek Instruments, Inc., Winooski, VT, USA). The experiments were repeated three times for each experimental group.

Cell colony formation assay. BMSCs were plated in six-well plates at 1,000 cells per well and allowed to attach overnight at $37^{\circ} \mathrm{C}$ with $5 \% \mathrm{CO}_{2}$. The culture medium was then replaced with L-DMEM with $10 \%$ FBS containing 10,20 or $30 \mu \mathrm{g} / \mathrm{ml}$
IDB for 14 days. The control group was treated with DMSO. The medium was changed at 3-day intervals. Finally, the cells were fixed with $4 \%$ paraformaldehyde for $30 \mathrm{~min}$ and stained with crystal violet for $20 \mathrm{~min}$. The cells were photographed and the number of colonies was counted for statistical analysis. The experiments were repeated three times for each experimental group.

Morphological analysis. The cells $\left(2 \times 10^{5}\right)$ were seeded in 24-well plates in L-DMEM containing $10 \% \mathrm{FBS}$ with $5 \% \mathrm{CO}_{2}$ at $37^{\circ} \mathrm{C}$. After overnight incubation, cells were cultured with different concentrations of IDB (10, 20 and $30 \mu \mathrm{g} / \mathrm{ml})$ for $24 \mathrm{~h}$ and morphological changes were observed using an inverted microscope under 40x magnification.

DAPI staining. The cells were seeded in 24-well plates at a density of 20,000 cells per well. After culture with IDB at $10-30 \mu \mathrm{g} / \mathrm{ml}$ for $24 \mathrm{~h}$, the cells were washed three times with PBS, then fixed with $4 \%$ paraformaldehyde for $20 \mathrm{~min}$ and washed again with PBS. DAPI staining solution (final concentration $5 \mu \mathrm{g} / \mathrm{ml}$ ) was added and cells were incubated in the dark at room temperature for $5 \mathrm{~min}$. The DAPI staining solution was removed and the cells were washed three times with PBS, 5 min per wash. The stained cells were observed directly under a fluorescence microscope. Three non-overlapping fields were randomly captured per dish. The experiments were repeated three times for each experimental group.

Cell cycle assay. BMSCs were plated in six-well plates at the third passage. After treatment with different concentrations of IDB $(10,20$ or $30 \mu \mathrm{g} / \mathrm{ml})$ for $24 \mathrm{~h}$, the BMSCs were harvested, washed twice using cold PBS and stained with propidium iodide (PI; Sigma-Aldrich; Merck KGaA, Darmstadt, Germany) for $30 \mathrm{~min}$ at $4^{\circ} \mathrm{C}$ in the dark. We then used flow cytometry as described above to determine the cell cycle. The experiments were repeated three times for each experimental group.

Apoptosis assay. Cells were seeded in six-well plates and incubated overnight. After treatment with IDB at 10,20 or $30 \mu \mathrm{g} / \mathrm{ml}$ for $24 \mathrm{~h}$, the cells were harvested, rinsed twice with cold PBS and incubated with PI and AnnexinV-FITC (BD Biosciences) for $20 \mathrm{~min}$ at room temperature. The stained cells were analyzed with flow cytometry as previously described. The experiments were performed in triplicate.

Transwell migration assay. The migration of BMSCs was tested in transwell chemotaxis chambers (Corning Incorporated, Corning, NY, USA). Briefly, after culture with IDB $(10,20$ or $30 \mu \mathrm{g} / \mathrm{ml})$ for $24 \mathrm{~h}, 3 \times 10^{4}$ cells were suspended in $200 \mu \mathrm{l}$ serum-free medium and added to the upper chamber. Next, $600 \mu \mathrm{l}$ of culture medium with $10 \%$ FBS was added to the lower chamber and the cells were incubated for $16 \mathrm{~h}$. BMSCs which remained on the top side of the membrane were wiped off with cotton swabs. Cells which had migrated to the bottom side of the membrane were fixed in $4 \%$ paraformaldehyde and stained with crystal violet. We observed the stained cells under a microscope and selected at least six fields for each group. The experiments were repeated in triplicate for each group. 

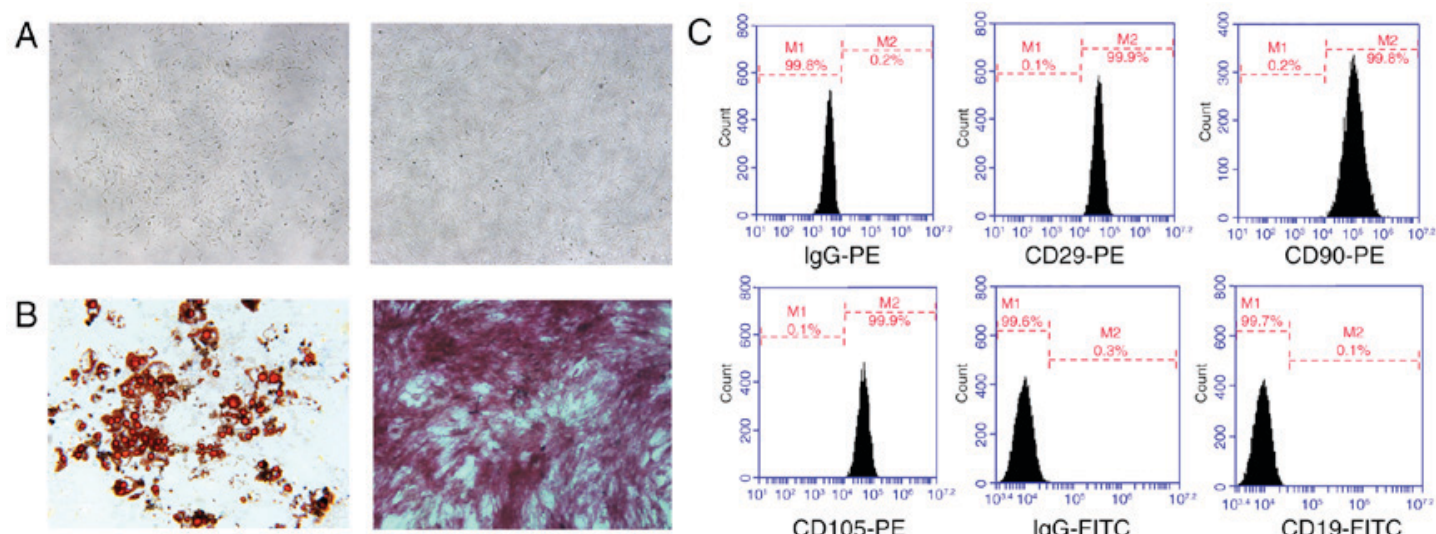

CD29-PE
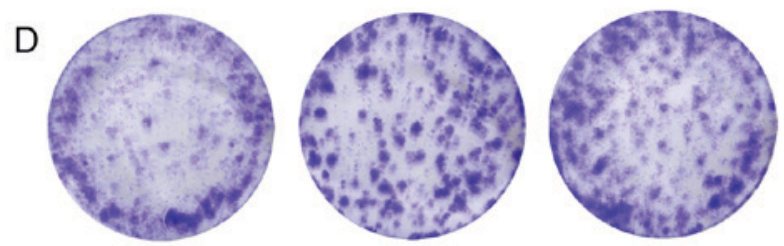

CD105-PE

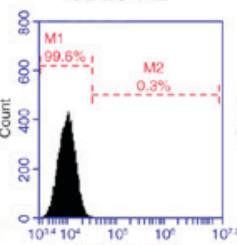

CD90-PE
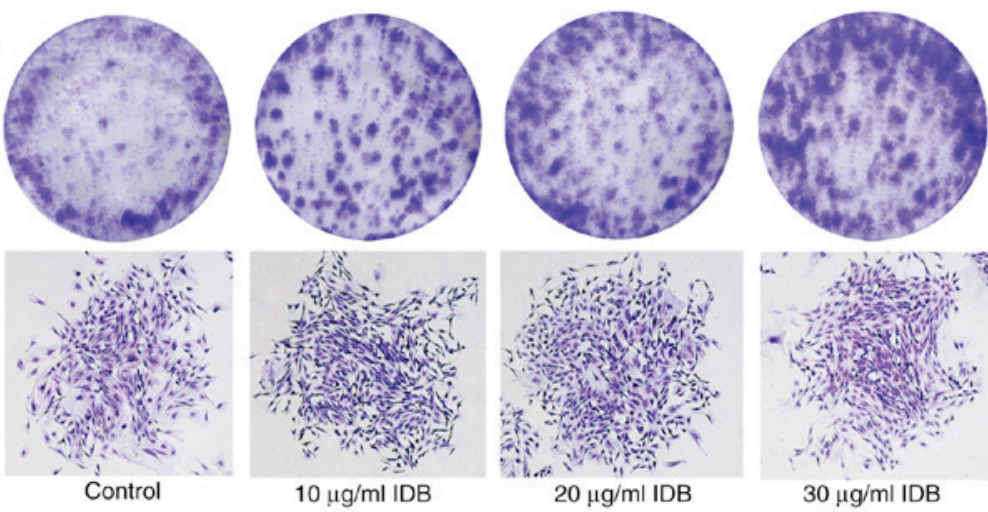

IgG-FITC
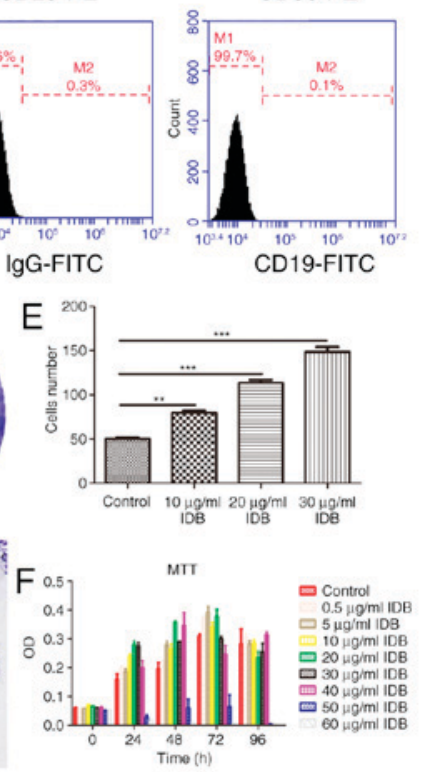

Figure 1. Characterization of BMSCs and the effect of IDB on cell proliferation. (A) Morphology of BMSCs. Polygonal, spindly and fibroblast-like cells in primary culture (magnification, $\mathrm{x} 40$ ). (B) BMSCs were induced towards adipogenic and osteogenic differentiation in the third generation shown by staining for Oil Red O (left panel) and alkaline phosphatase (right panel) (magnification, x100). (C) Surface markers identified by fluorescence-activated cell sorting analysis. BMSCs were positive for CD29, CD90 and CD105, and negative for CD19. (D) Effect of IDB on colony formation. Images of the cell colony formation assay and the morphology of colonies (magnification, $\mathrm{x} 40$ ). (E) Histogram depicting the number of colonies. (F) Viability of BMSCs following treatment with IDB. All cells were incubated with increasing concentrations of IDB $(0.5-60 \mu \mathrm{g} / \mathrm{ml})$ for $0-96 \mathrm{~h}$ and cell viability was determined with MTT assay. All data are presented as the mean \pm standard deviation. ${ }^{* *} \mathrm{P}<0.01$ and ${ }^{* * * *} \mathrm{P}<0.001$, as indicated. BMSCs, bone marrow-mesenchymal stem cells; IDB, Idebenone; MTT, 3-(4,5-dimethylthiazol-2-yl-)-2,5-diphenyl tetrazolium bromide.

Senescence-associated $\beta$-galactosidase staining. Expression of senescence-associated $\beta$-galactosidase (SA- $\beta$-gal) in BMSCs was detected using a SA- $\beta$-gal staining kit (Beyotime Institute of Biotechnology, Haimen, China). The cells were washed with PBS, fixed for 15 min using $4 \%$ formaldehyde and washed again with PBS. Subsequently, SA- $\beta$-gal stain solution was added and the plates were placed into a $\mathrm{CO}_{2}$-free chamber. The percentage of blue-stained SA- $\beta$-gal-positive cells was counted in three random fields. Experiments were repeated at least three times.

Western blot analysis. BMSCs were cultured in IDB at $10-30 \mu \mathrm{g} / \mathrm{ml}$ for $24 \mathrm{~h}$ after cell density reached about $80 \%$. The total protein from the BMSCs was obtained with RIPA lysis buffer containing protease inhibitor cocktail (Invitrogen, Carlsbad, CA, USA). The protein concentration of each sample was determined with a BCA protein assay kit (Invitrogen, Carlsbad, CA, USA). Proteins from each group were subjected to separation by SDS-polyacrylamide gel electrophoresis and then transferred onto polyvinylidene fluoride membranes. After blocking with 5\% non-fat milk for $1 \mathrm{~h}$, the membranes were incubated with monoclonal antibodies (mouse anti-rat) PCNA, cyclinD1, cyclinD3, Stat-3, Nanog, Oct-4, vimentin, E-cadherin, N-cadherin, Bcl-2, Bax, cleaved caspase-3 and monoclonal antibodies (rat anti-mouse) $\alpha$-SMA at $4^{\circ} \mathrm{C}$ overnight. The membranes were washed with Tris-buffered saline/Tween three times and incubated with the appropriate secondary antibodies at $37^{\circ} \mathrm{C}$ for $1 \mathrm{~h}$. The signals were visualized with Luminata Crescendo Western HRP substrate (Millipore, Billerica, MA, USA). All experiments were repeated at least three times.

Statistical analysis. Experiments were performed in triplicate and repeated at least three times independently. All data were expressed as means \pm standard deviation (SD). Statistically significant differences between groups were assessed by analysis of variance using the GraphPad PrismV5.0 software program (GraphPad, San Diego, CA, USA). $\mathrm{P}<0.05$ was considered to indicate a statistically significant difference.

\section{Results}

Characterization of BMSCs and the effect of IDB on cell proliferation and cell formation. In primary culture, BMSCs displayed a polygonal spindly shape and contained one nucleus. After 7-10 days, they presented with a long spindle-shape and began to form colonies (Fig. 1A). The cells were induced to undergo adipogenic or osteogenic differentiation in the third generation, as shown by Oil Red O and alkaline phosphatase 


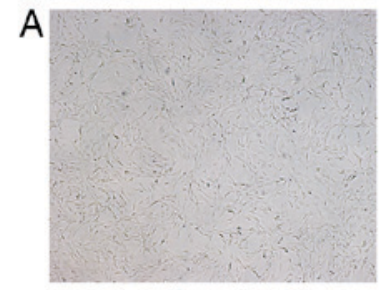

Control

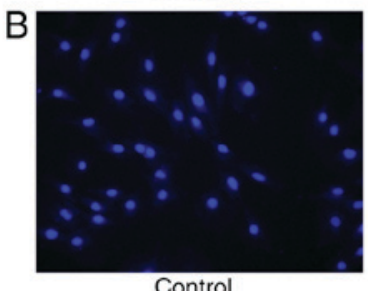

Control

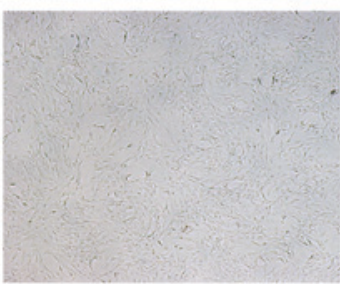

$10 \mu \mathrm{g} / \mathrm{ml}$ IDB

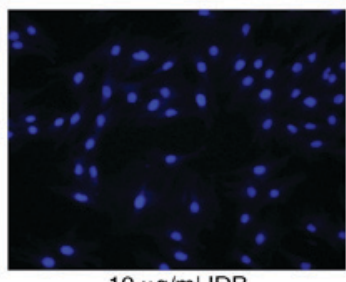

$10 \mu \mathrm{g} / \mathrm{ml}$ IDB

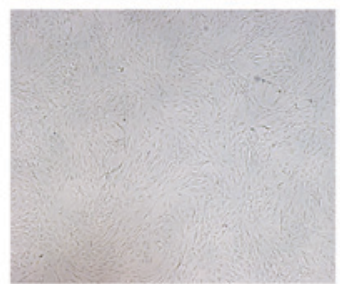

$20 \mu \mathrm{g} / \mathrm{ml}$ IDB

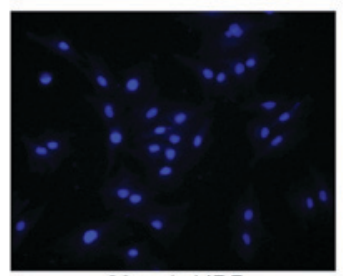

$20 \mu \mathrm{g} / \mathrm{ml} \mathrm{IDB}$ $20 \mu \mathrm{g} / \mathrm{ml}$ IDB

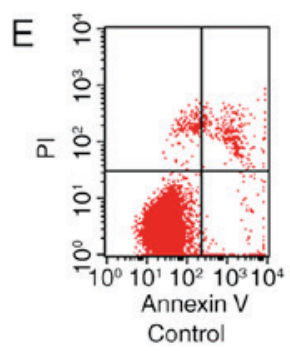

$10 \mu \mathrm{g} / \mathrm{ml}$ IDB

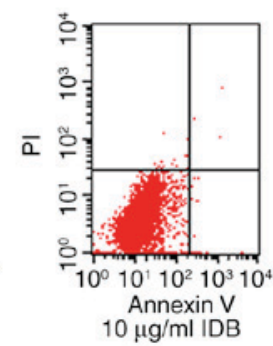

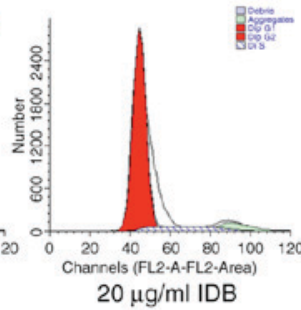

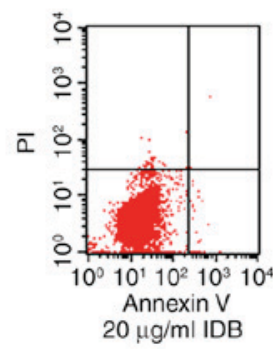

焉
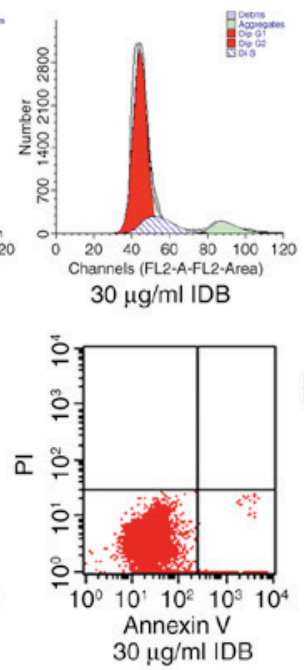

$30 \mu \mathrm{g} / \mathrm{ml}$ IDB

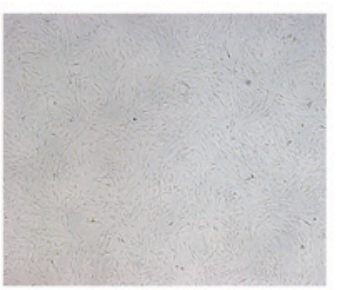

$30 \mu \mathrm{g} / \mathrm{ml} \mathrm{IDB}$

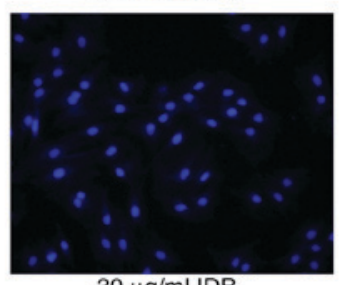

$30 \mu \mathrm{g} / \mathrm{ml}$ IDB
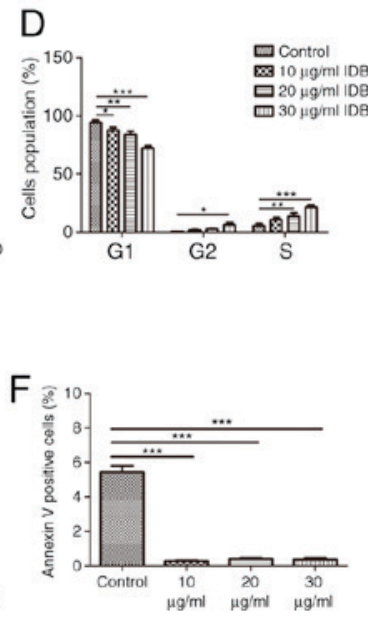

Figure 2. Effect of IDB on morphology, cell cycle and apoptosis of BMSCs. (A) The morphology of BMSCs changed in a dose-dependent manner following exposure to IDB (magnification, x40). (B) Nuclear analysis of BMSCs with DAPI staining (magnification, x100). (C) Effect of IDB on the cell cycle. (D) DNA content analysis indicated that the percentage of cells in the G2 and S phases were increased when compared with the control group. (E) Cells were stained with Annexin V-fluorescein isothiocyanate/propidium iodide and analyzed using flow cytometry to determine the cell apoptosis. (F) Percentage of Annexin V-positive cells are shown in the histogram. All data are displayed as the mean \pm standard deviation. ${ }^{*} \mathrm{P}<0.05^{* *} \mathrm{P}<0.01$ and ${ }^{* * * *} \mathrm{P}<0.001$, as indicated. BMSCs, bone marrow-mesenchymal stem cells; IDB, Idebenone.

staining, respectively (Fig. 1B). According to flow cytometric analysis, BMSCs were positive for CD29, CD90 and CD105, but negative for CD19 (Fig. 1C).

The results of cell colony formation assays showed a dose-related increase in BMSC proliferation after incubation in increasing concentrations of IDB. Cell morphology changed from a broad flattened to a long spindle shape. At lower concentrations $(10 \mu \mathrm{g} / \mathrm{ml})$, IDB also had an obvious effect on the number and the morphology of colonies (Fig. 1D). Statistical analysis showed a significant increase in cell number between the control group and each treatment group with increasing IDB concentration (Fig. 1E).

MTT assays indicated that the viability of BMSCs in each treatment group was increased in a dose and time-dependent manner. At IDB concentrations of 10, 20 and $30 \mu \mathrm{g} / \mathrm{ml}$, proliferative activity in the experimental groups was significantly higher than in the control group, but higher concentrations had no further effect on proliferation. Therefore, we selected the concentrations of 10,20 and $30 \mu \mathrm{g} / \mathrm{ml}$ for the following experiments (Fig. 1F).
The effect of IDB on biological characteristics of BMSCs. In the experimental groups, we observed that reticular formations of BMSCs appeared gradually and that the cells were tightly connected. The morphological shape of the cells became more like a slender spindle with increasing drug concentration. However, these changes were not found in the control group (Fig. 2A). The protective effects of IDB were further confirmed with DAPI staining. The cells were smaller and thinner in contrast to the DMSO group. In the IDB group, chromatin morphological changes were slightly improved (Fig. 2B). Using PI staining, the cell cycle in the presence of IDB was characterized by flow cytometric analysis. The results demonstrated that the cells in G1 phase in the treatment groups were marginally decreased while cells in $\mathrm{S}$ phase were slightly increased when compared with the control group. This suggested that IDB could promote the transformation of cells from $\mathrm{G} 1$ to $\mathrm{S}$ phase as shown by histogram analysis indicating statistically significant differences between the control and experimental groups (Fig. 2C, D). Considering the important role of IDB in cell apoptosis, we also used flow cytometry 


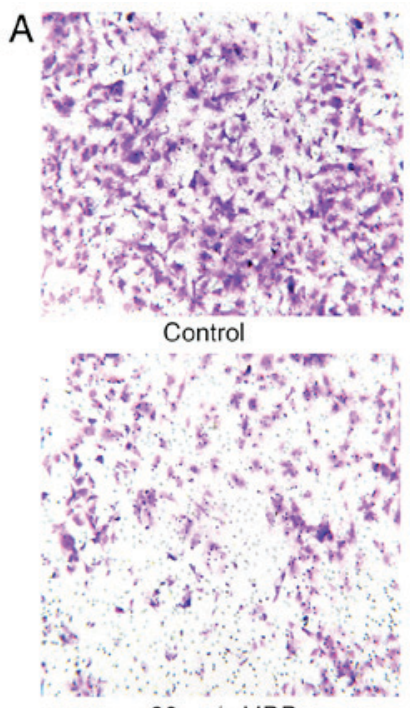

$20 \mu \mathrm{g} / \mathrm{ml} \mathrm{IDB}$

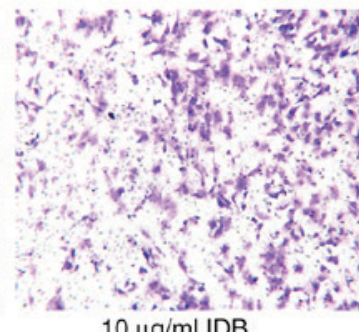
$10 \mu \mathrm{g} / \mathrm{ml} \mathrm{IDB}$

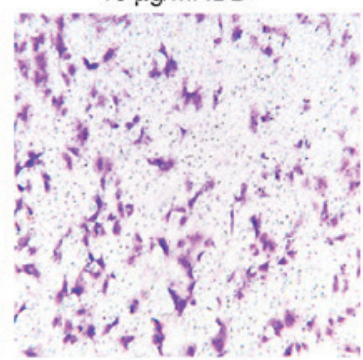

$30 \mu \mathrm{g} / \mathrm{ml}$ IDB

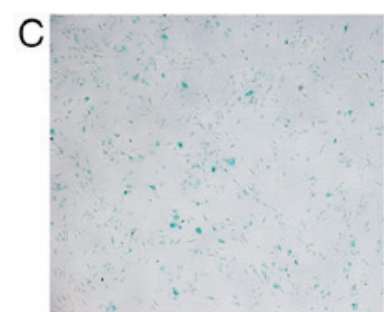

Control

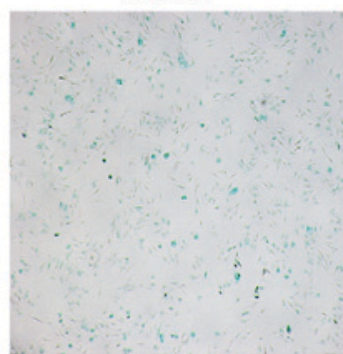

$20 \mu \mathrm{g} / \mathrm{ml} \mathrm{IDB}$

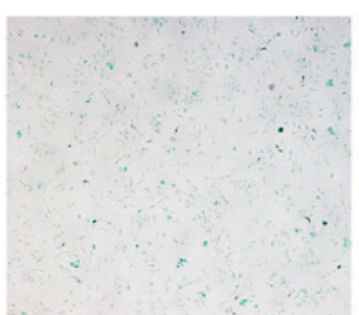

$10 \mu \mathrm{g} / \mathrm{ml}$ IDB

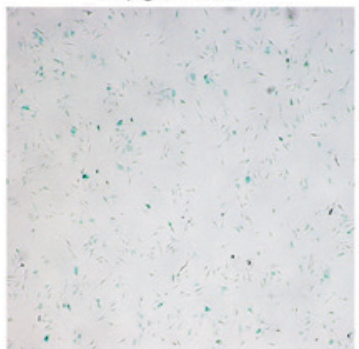

$30 \mu \mathrm{g} / \mathrm{ml}$ IDB
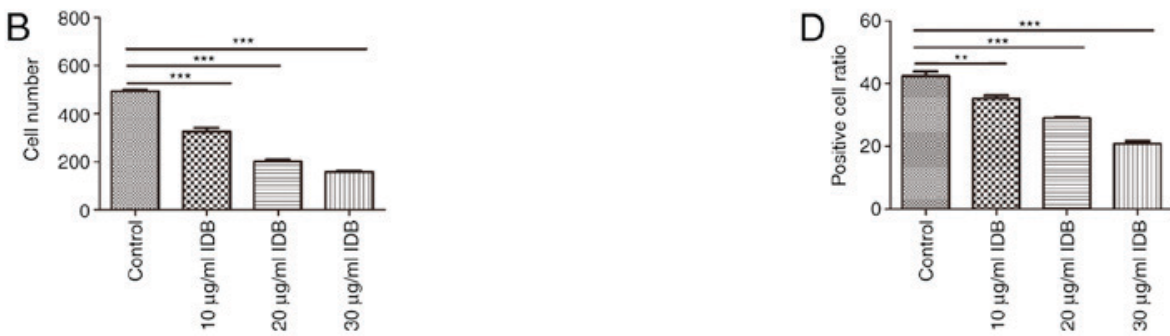

Figure 3. Effect of IDB on cell migration and SA- $\beta$-gal staining. (A) Representative images from transwell migration assays (magnification, $\mathrm{x} 100$ ). (B) Histogram showing the number of migrated control and IDB-treated cells. (C) SA- $\beta$-galactosidase staining (magnification, $x 100$ ). (D) The control group had a greater number of senescent cells when compared with the experimental groups. All data are displayed as the mean \pm standard deviation. ${ }^{* *} \mathrm{P}<0.01$ and ${ }^{* * *} \mathrm{P}<0.001$, as indicated. BMSCs, bone marrow-mesenchymal stem cells; IDB, Idebenone; SA- $\beta$-gal, senescence-associated $\beta$-galactosidase.

to detect apoptosis after treatment with IDB. The number of apoptotic cells in the IDB-treated groups was significantly less than in the DMSO group (Fig. 2E, F). Therefore, it appears that low concentrations of IDB may have an anti-apoptotic effect.

IDB suppressed cell migration and alleviated cell senescence. To determine whether the migratory ability of BMSCs was affected by IDB, we cultured the cells with increasing concentrations of IDB for $24 \mathrm{~h}$. The results of cell migration assays indicated that IDB inhibited the migration of BMSCs when compared with the control group (Fig. 3A). Statistical analysis demonstrated a significant dose-related reduction in cell numbers after IDB exposure (Fig. 3B). The results of SA- $\beta$-gal staining showed that the experimental groups had fewer senescent cells and that senescence was decreased in a dose-dependent manner (Fig. 3C). Statistical analysis demonstrated that IDB significantly reduced the number of senescent cells (Fig. 3D).

Western blot analysis of the effects of IDB on BMSCs. To investigate the underlying mechanisms of IDB on BMSCs, we performed western blot experiments. We discovered that IDB reduced the expression of $\mathrm{N}$-cadherin, vimentin and $\alpha$-SMA but enhanced the expression of E-cadherin in vitro. These results are consistent with the role of these proteins in IDB-induced inhibition of migratory behavior in BMSCs (Fig. 4A-E). We also found that expression of Stat-3, Nanog and
Oct-4 were increased after IDB exposure in a dose-dependent manner (Fig. 4F-I). Similarly, the expression of PCNA, cyclinD1 and cyclinD3 were increased after IDB treatment (Fig. 4J-M). Finally, we examined the important functions of Bcl-2, Bax and cleaved caspase- 3 in the inhibition of cell apoptosis. Quantitative analysis revealed that IDB distinctly increased the ratio of $\mathrm{Bcl}-2$ to $\beta$-actin while decreasing the ratio of Bax to $\beta$-actin (Fig. 4N-P). Moreover, the expression of cleaved caspase-3 protein, which is a key factor in the execution of apoptosis, was significantly suppressed as shown by statistical analysis (Fig. 4N-Q).

\section{Discussion}

Recently, IDB has been used as an antioxidant to resist skin damage in anti-aging cosmetics (22). Compared with common cosmetic antioxidants such as vitamin E, vitamin $\mathrm{C}$ and coenzyme Q10, IDB has shown to be the most effective antioxidant in global assessments (23). As is well known, antioxidant therapy is widely used to improve cardiac and neurological functions, especially for Friedreich ataxia patients $(24,25)$. As the most effective antioxidant, IDB is a potential therapeutic agent for a great number of diseases including mitochondrial diseases and Alzheimer's disease $(24,25)$.

Currently, people are paying more attention to the use of anti-oxidative cosmetics to improve quality of life. IDB, as a 
A
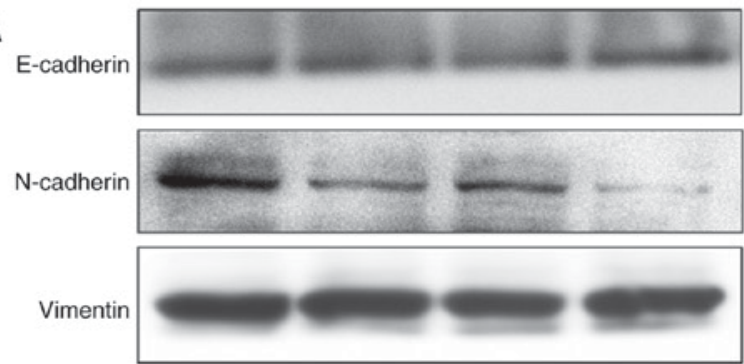

$\alpha-S M A$

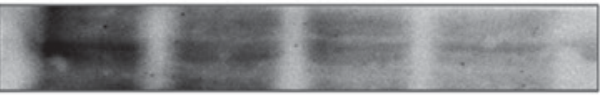

$\beta$-actin

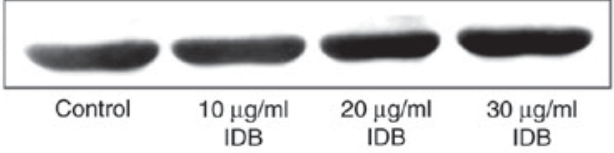

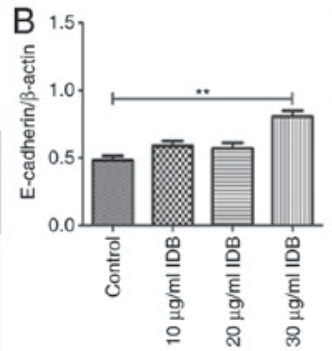

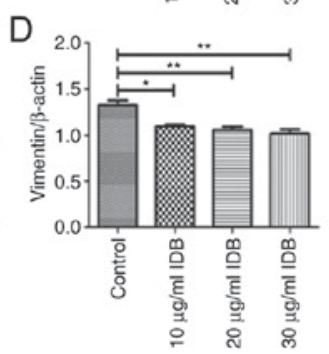

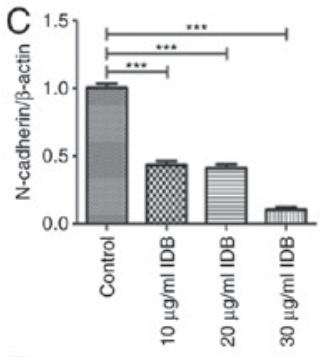

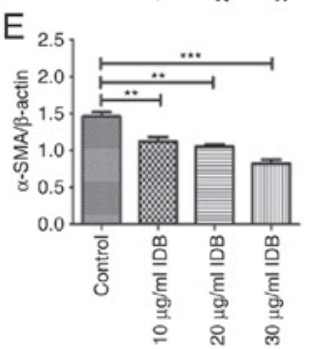

$\mathrm{F}$

Stat-3

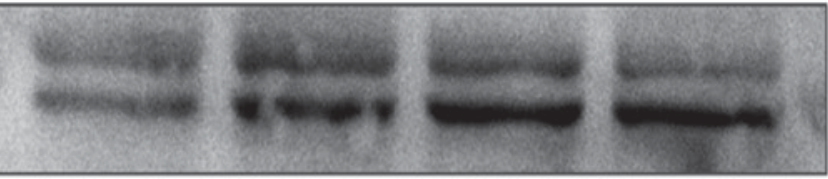

Nanog
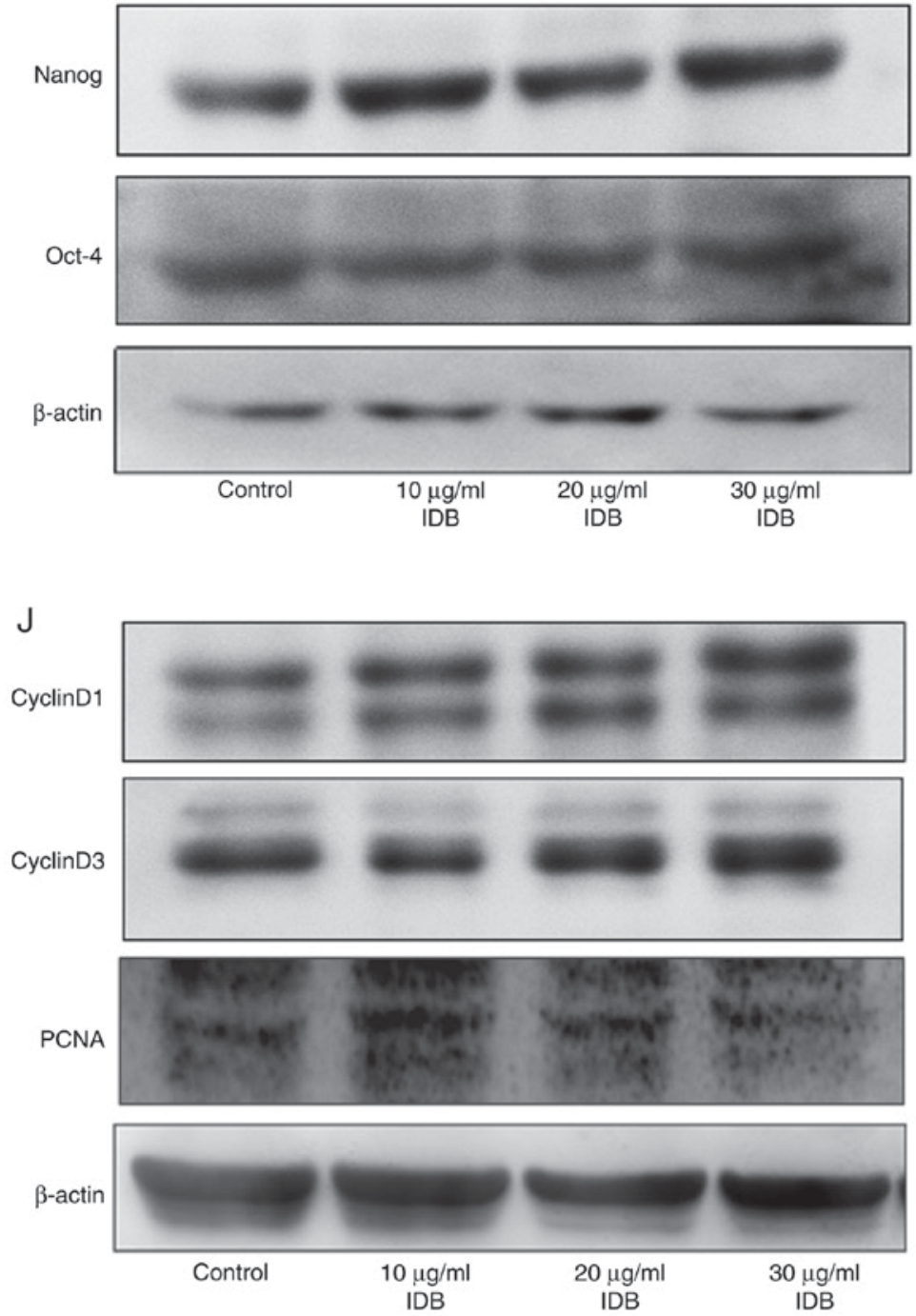
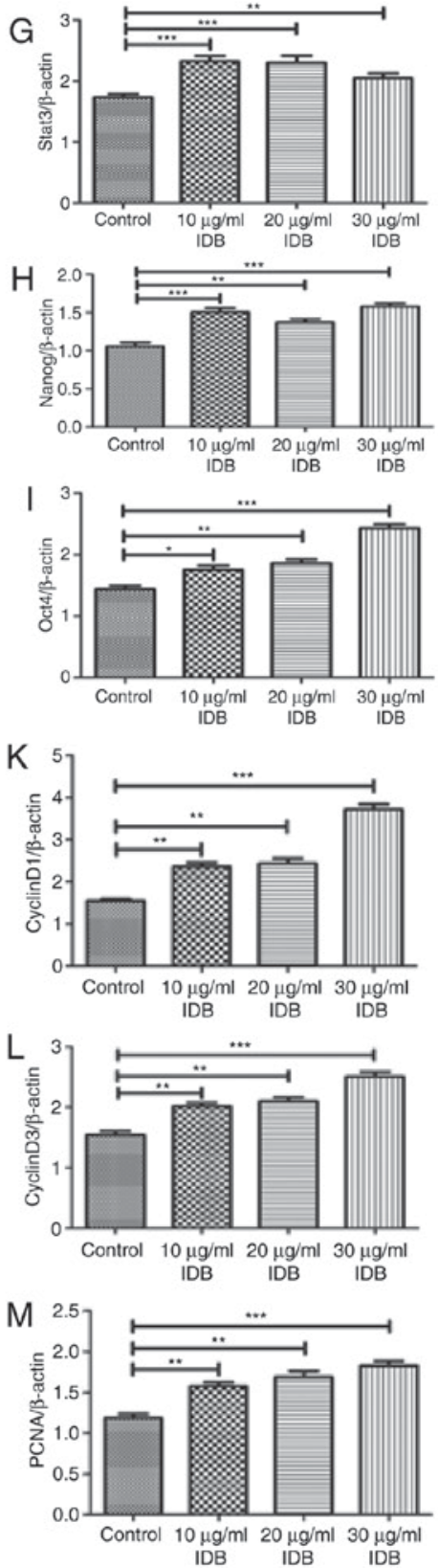

Figure 4. Effect of IDB on protein expression examined by western blotting and gray scanning analysis. (A) The expression of endothelial mesenchymal transition associated proteins and the results of statistical analyses for (B) E-cadherin, (C) N-cadherin, (D) vimentin and (E) $\alpha$-SMA. (F) The protein expression of the stem cell markers (G) Stat-3, (H) Nanog and (I) Oct-4. (J) The protein expression of (K) cyclinD1, (L) cyclinD3 and (M) PCNA. 
$\mathrm{N}$
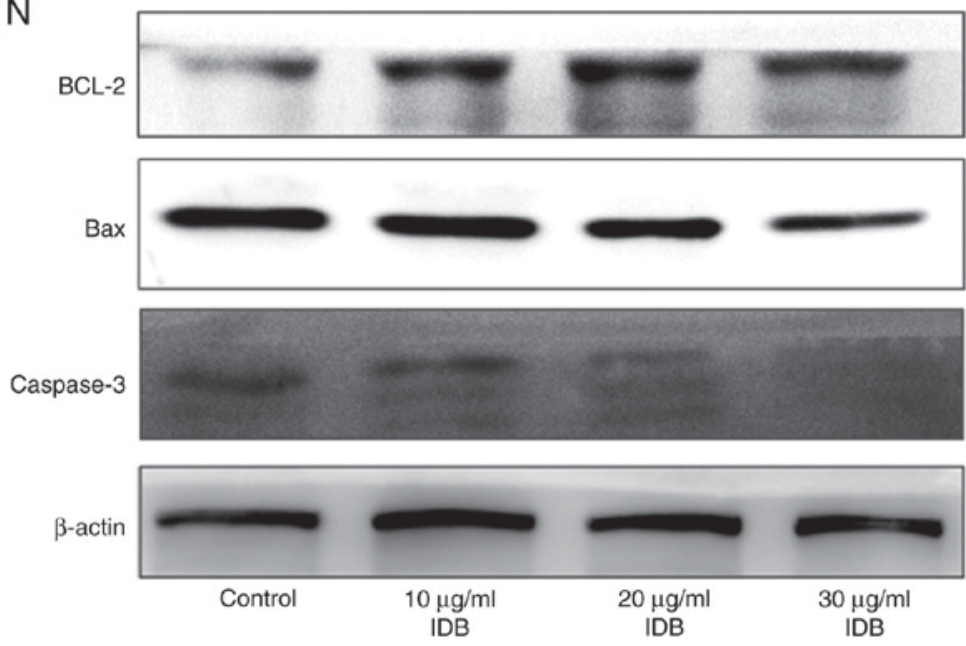

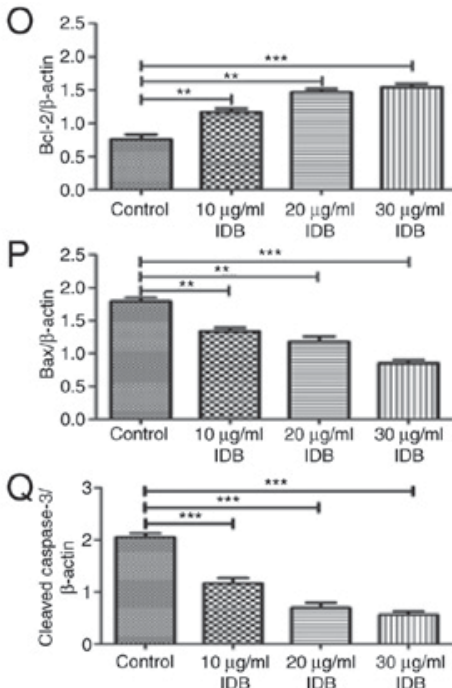

Figure 4. Continued. (N) The expression of proteins involved in apoptotic signaling including $(\mathrm{O}) \mathrm{Bcl}-2$, (P) Bax and (Q) cleaved caspase-3. $\beta$-actin was used as an internal reference. All data are displayed as the mean \pm standard deviation. ${ }^{*} \mathrm{P}<0.05{ }^{* *} \mathrm{P}<0.01$ and ${ }^{* * *} \mathrm{P}<0.001$, as indicated. IDB, Idebenone; PCNA, proliferating cell nuclear antigen; SMA, smooth muscle actin; Stat-3, signal transducer and activator of transcription 3; Oct-4, octamer-binding transcription factor 4; Bcl-2, B-cell lymphoma 2; Bax, Bcl-2-associated X protein.

kind of exogenous antioxidants, has the effect of eliminating oxygen free radicals, and exerts anti-aging, anti-wrinkle and other effects. IDB acts as a novel antioxidant that can prevent the formation of reactive oxygen species and reactive radicals (26-28).

However, studies examining whether IDB as a drug or cosmetic contains harmful substances which may cause skin edema, itching and other damage to the human body have been inconclusive.

In our study, we aimed to investigate the biological effect of IDB on BMSCs, and its underlying mechanisms. From MTT and cell colony formation assays, we found that IDB at a concentration between 10 and $30 \mu \mathrm{g} / \mathrm{ml}$ obviously promoted cell proliferation and cell colony formation. We then performed DAPI staining and examined SA- $\beta$-gal staining as a marker of cellular senescence. By comparing the treatment group with the control group, we discovered that the chromatin morphological changes were restrained and cellular senescence was reduced as well after exposure to IDB. We also performed cell apoptosis and cell cycle assays. Compared with the control group, the percentage of cells in G1 phase after IDB treatment was marginally decreased, the percentage of cells in S phase was increased and the proportion of apoptotic cells was significantly reduced. These results suggest that IDB may inhibit cell aging and maintain the immature state of cells.

More recent studies have shown that BMSCs secrete a large amount of cytokines and reduce the immune response (29-32). Therefore, we examined the effect of IDB on a number of these cytokines. Combining the expression of endothelial-mesenchymal transition-related proteins with cell migration experiments, we determined that IDB in low concentrations dramatically suppressed the migration of BMSCs. Our western blot analysis also suggested that IDB could enhance the expression of stem cell markers such as Nanog and Oct-4 and increase the expression of cell cycle-related proteins including cyclinD1 and cyclinD3. In addition, IDB also upregulated the expression of Stat-3 and PCNA. Finally, we demonstrated that IDB prevented cellular damage and inhibited cell apoptosis.

Previous studies have revealed that apoptosis plays an important role in regulating stem cell-dependent regeneration. Thus, it was necessary to investigate the mechanisms underlying the anti-apoptotic effects of IDB. Western blot analysis was performed to determine the expression levels of Bcl-2, Bax and cleaved caspase-3, which are well known as critical modulators of apoptosis. In our study, we found that protein levels of cleaved caspase- 3 and Bax were significantly decreased following treatment with IDB at low concentrations. In contrast, the level of Bcl-2 was significantly increased, further validating the apoptotic pathway.

In conclusion, IDB has a significant protective effect on BMSCs involving apoptosis, migration, proliferation and the expression of related proteins. Our research has demonstrated that IDB at low concentrations in drugs and cosmetics may have no toxic impact on BMSCs. However, further investigations should be designed to clarify the downstream genes which play important roles in cell apoptosis and to determine if IDB affects other aspects of BMSCs.

\section{Acknowledgements}

The present study was supported by 2015 Jiangsu Province Distinguished Professor Fund.

\section{References}

1. Dong KK, Damaghi N, Kibitel J, Canning MT, Smiles KA and Yarosh DB: A comparison of the relative antioxidant potency of L-ergothioneine and idebenone. J Cosmet Dermatol 6: 183-188, 2007.

2. Schulz JB, Di Prospero NA and Fischbeck K: Clinical experience with high-dose IDB in Friedreich ataxia. J Neurol 256 (Suppl 1): S42-S45, 2009

3. Ahmed MA: Neuroprotective effects of idebenone against pilocarpine-induced seizures: Modulation of antioxidant status, DNA damage and $\mathrm{Na}(+), \mathrm{K}(+)$-ATPase activity in rat hippocampus. Neurochem Res 39: 394-402, 2014. 
4. Gutzmann H, Kühl KP, Hadler D and Rapp MA: Safety and efficacy of idebenone versus tacrine in patients with Alzheimer's disease: Results of a randomized, double-blind, parallel-group multicenter study. Pharmacopsychiatry 35: 12-18, 2002.

5. Parkinson MH, Schulz JB and Giunti P: Co-enzyme Q10 and idebenone use in Friedreich's ataxia. J Neurochem 126 (Suppl 1): S125-S141, 2013.

6. Rustin P, von Kleist-Retzow JC, Chantrel-Groussard K, Sidi D, Munnich A and Rötig A: Effect of idebenone on cardiomyopathy in Friedreich's ataxia: A preliminary study. Lancet 354: 477-479, 1999.

7. Rustin P, Rötig A, Munnich A and Sidi D: Heart hypertrophy and function are improved by idebenone in Friedreich's ataxia. Free Radic Res 36: 467-469, 2002.

8. Artuch R, Aracil A, Mas A, Colomé C, Rissech M, Monrós E and Pineda M: Friedreich's ataxia: Idebenone treatment in early stage patients. Neuropediatrics 33: 190-193, 2002.

9. Hausse AO, Aggoun Y, Bonnet D, Sidi D, Munnich A, Rötig A and Rustin P: idebenone and reduced cardiac hypertrophy in Friedreich's ataxia. Heart 87: 346-349, 2002.

10. La Pean A, Jeffries N, Grow C, Ravina B and Di Prospero NA: Predictors of progression in patients with Friedreich ataxia. Mov Disord 23: 2026-2032, 2008.

11. Di Prospero NA, Baker A, Jeffries $\mathrm{N}$ and Fischbeck $\mathrm{KH}$ Neurological effects of high-dose idebenone in patients with Friedreich's ataxia: A randomised, placebo-controlled trial. Lancet Neurol 6: 878-886, 2007.

12. Tonon $\mathrm{C}$ and Lodi R: Idebenone in Friedreich's ataxia. Expert Opin Pharmacother 9: 2327-2337, 2008.

13. Pineda M, Arpa J, Montero R, Aracil A, Domínguez F, Galván M, Mas A, Martorell L, Sierra C, Brandi N, et al: idebenone treatment in paediatric and adult patients with Friedreich ataxia: Long-term follow-up. Eur J Paediatr Neurol 12: 470-475, 2008.

14. Riazi A, Cano SJ, Cooper JM, Bradley JL, Schapira AH and Hobart JC: Coordinating outcomes measurement in ataxia research: Do some widely used generic rating scales tick the boxes? Mov Disord 21: 1396-1403, 2006.

15. Zhu GR, Zhou XY, Lu H, Zhou JW, Li AP, Xu W, Li JY and Wang CY: Human bone marrow mesenchymal stem cells express multiple hematopoietic growth factors. Zhongguo Shi Yan Xue Ye Xue Za Zhi 11: 115-119, 2003 (In Chinese)

16. da Silva Meirelles L, Sand TT, Harman RJ, Lennon DP and Caplan AI: MSC frequency correlates with blood vessel density in equine adipose tissue. Tissue Eng Part A 15: 221-229, 2009.

17. Antunes MA, Abreu SC, Cruz FF, Teixeira AC, Lopes-Pacheco M, Bandeira E, Olsen PC, Diaz BL, Takyia CM, Freitas IP, et al: Effects of different mesenchymal stromal cell sources and delivery routes in experimental emphysema. Respir Res 15: 118, 2014.

18. Barry FP and Murphy JM: Mesenchymal stem cells: Clinical applications and biological characterization. Int J Biochem Cel Biol 36: 568-584, 2004.
19. Yang ZX, Han ZB, Ji YR, Wang YW, Liang L, Chi Y, Yang SG, Li LN, Luo WF, Li JP, et al: CD106 identifies a subpopulation of mesenchymal stem cells with unique immunomodulatory properties. PLoS One 8: e59354, 2013.

20. Burk J, Gittel C, Heller S, Pfeiffer B, Paebst F, Ahrberg AB and Brehm W: Gene expression of tendon markers in mesenchymal stromal cells derived from different sources. BMC Res Notes 7: $826,2014$.

21. Xu H, Qian H, Zhu W, Zhang X, Yan Y, Mao F, Wang M, $\mathrm{Xu} \mathrm{H}$ and $\mathrm{Xu}$ W: Mesenchymal stem cells relieve fibrosis of Schistosoma japonicum-induced mouse liver injury. Exp Biol Med (Maywood) 237: 585-592, 2012.

22. Farris P: Idebenone, green tea, and Coffeeberry extract: New and innovative antioxidants. Dermatol Ther 20: 322-329, 2007.

23. McDaniel DH, Neudecker BA, DiNardo JC, Lewis JA II and Maibach HI: Idebenone: A new antioxidant-Part I. Relative assessment of oxidative stress protection capacity compared to commonly known antioxidants. J Cosmet Dermatol 4: 10-17, 2005.

24. Haginoya K, Miyabayashi S, Kikuchi M, Kojima A, Yamamoto K, Omura K, Uematsu M, Hino-Fukuyo N, Tanaka S and Tsuchiya S: Efficacy of idebenone for respiratory failure in a patient with Leigh syndrome: A long-term follow-up study. J Neurol Sci 278: 112-114, 2009.

25. Meier $\mathrm{T}$ and Buyse $\mathrm{G}$ : Idebenone: An emerging therapy for Friedreich ataxia. J Neurol 256 (Suppl 1): S25-S30, 2009.

26. Zs -Nagy I: Chemistry, toxicology, pharmacology and pharmacokinetics of idebenone: A review. Arch Gerontol Geriatr 11: 177-186, 1990.

27. Gillis JC, Benefield P and McTavish D: Idebenone. A review of its pharmacodynamic and pharmacokinetic properties, and therapeutic use in age-related cognitive disorders. Drugs Aging 5: 133-152, 1994

28. Suno M and Nagaoka A: Inhibition of lipid peroxidation by a novel compound (CV-2619) in brain mitochondria and mode of action of the inhibition. Biochem Biophys Res Commun 125: 1046-1052, 1984.

29. Deng W, Han Q, Liao L, You S, Deng H and Zhao RC: Effects of allogeneic bone marrow-derived mesenchymal stem cells on T and B lymphocytes from BXSB mice. DNA Cell Biol 24: 458-463, 2005 .

30. Corcione A, Benvenuto F, Ferretti E, Giunti D, Cappiello V, Cazzanti F, Risso M, Gualandi F, Mancardi GL, Pistoia V and Uccelli A: Human mesenchymal stem cells modulate B-cell functions. Blood 107: 367-372, 2006

31. Yin Z, Chen D, Hu F, Ruan Y, Li J, Wang L, Xiang Y, Xie L, Wang X, Ichim TE, et al: Cotransplantation with xenogenetic neonatal porcine sertoli cells significantly prolongs islet allograft survival in nonimmunosuppressive rats. Transplantation 88 : 339-345, 2009

32. Dufour JM, Dass B, Halley KR, Korbutt GS, Dixon DE and Rajotte RV: Sertoli cell line lacks the immunoprotective properties associated with primary Sertoli cells. Cell Transplant 17: $525-534,2008$ 